\title{
Asymmetric tri-bi-maximal mixing and residual symmetries
}

\author{
Salvador Centelles Chuliá ${ }^{1, *}$ and Andreas Trautner ${ }^{2, \dagger}$ \\ ${ }^{1}$ AHEP Group, Institut de Física Corpuscular - \\ CSIC-Universitat de València, Parc Científic de Paterna. \\ C/ Catedrático José Beltrán, 2 E-46980 Paterna (Valencia) - SPAIN \\ ${ }^{2}$ Max-Planck-Institut für Kernphysik, Saupfercheckweg 1, 69117 Heidelberg, Germany
}

Asymmetric tri-bi-maximal mixing is a recently proposed, grand unified theory (GUT) based, flavor mixing scheme. In it, the charged lepton mixing is fixed by the GUT connection to down-type quarks and a $\mathcal{T}_{13}$ flavor symmetry, while neutrino mixing is assumed to be tri-bi-maximal (TBM) with one additional free phase. Here we show that this additional free phase can be fixed by the residual flavor and CP symmetries of the effective neutrino mass matrix. We discuss how those residual symmetries can be unified with $\mathcal{T}_{13}$ and identify the smallest possible unified flavor symmetries, namely $\left(\mathbb{Z}_{13} \times \mathbb{Z}_{13}\right) \rtimes \mathrm{D}_{12}$ and $\left(\mathbb{Z}_{13} \times \mathbb{Z}_{13}\right) \rtimes \mathrm{S}_{4}$. Sharp predictions are obtained for lepton mixing angles, $\mathrm{CP}$ violating phases and neutrinoless double beta decay.

\section{ASYMMETRIC TRI-BI-MAXIMAL MIXING}

Still there is no compelling theory of the observed masses and mixings of elementary fermions. The only way to do away with the large number of apparently independent but correlated parameters is symmetry. Especially promising is the concept of grand unification, where the disconnected quark and lepton Yukawa couplings of the Standard Model are consolidated. This immediately raises the question why the observed mixing in the quark and lepton sectors would be so different. A straightforward explanation could be that a GUT induced similarity in the down-type quark and charged lepton mixings, in the spirit of "quark-lepton complementarity" [1-3], is distorted by the subtle mass generation of neutrinos. In this way, highly symmetric patterns for the neutrino mixing, such as TBM mixing $[4,5]$, would suffer a "Cabbibo haze" [6-8], i.e. corrections stemming from the charged fermion sector [9-11]. This could reconcile a high symmetry in neutrino mixing with the somewhat off-symmetric observed values, especially for the large $\theta_{13}$.

However, pure CKM-type mixing for the charged leptons together with TBM for the

*Electronic address: salcen@ific.uv.es

${ }^{\dagger}$ Electronic address: trautner@mpi-hd.mpg.de 
neutrino sector is observationally excluded. In fact, even for less symmetric patterns it was found that symmetric textures for the charged-lepton Yukawa matrix do not work [12]. It was therefore suggested to adopt a certain asymmetric texture for the charged lepton sector [13]. In a concrete realization, the charged left-handed lepton mixing matrix then was found to be of the form $[13,14]$

$$
U_{c \ell}=\left(\begin{array}{ccc}
1-\left(\frac{1}{18}+\frac{2}{9 A^{2}}\right) \lambda^{2} & \frac{1}{3} \lambda & \frac{2}{3 A} \lambda \\
-\frac{1}{3} \lambda & 1-\frac{1}{18} \lambda^{2} & A \lambda^{2} \\
-\frac{2}{3 A} \lambda & -\left(A+\frac{2}{9 A}\right) \lambda^{2} & 1-\frac{2}{9 A^{2}} \lambda^{2}
\end{array}\right)+\mathcal{O}\left(\lambda^{3}\right)
$$

where $\lambda=0.22453(44)$ and $A=0.836(15)$ are the usual quark sector Wolfenstein parameters [15]. Together with a "complex-TBM" (cTBM) neutrino mixing matrix, defined as

$$
U_{\nu}=U_{\mathrm{TBM} \delta}:=\left(\begin{array}{ccc}
\sqrt{\frac{2}{3}} & \frac{1}{\sqrt{3}} & 0 \\
-\frac{1}{\sqrt{6}} & \frac{1}{\sqrt{3}} & \frac{1}{\sqrt{2}} \\
\frac{\mathrm{e}^{\mathrm{i} \delta}}{\sqrt{6}} & -\frac{\mathrm{e}^{\mathrm{i} \delta}}{\sqrt{3}} & \frac{\mathrm{e}^{\mathrm{i} \delta}}{\sqrt{2}}
\end{array}\right),
$$

the Pontecorvo-Maki-Nakagawa-Sakata (PMNS) lepton mixing matrix would be given by

$$
U_{\text {lep }}=U_{c l}^{\dagger} U_{\nu}
$$

Since $U_{c \ell}$ is fixed by the GUT connection to quarks and the reproduction of the CKM matrix, no residual symmetry is allowed for the charged lepton mixing. In contrast, neutral lepton mixing in this framework is less constrained and it is possible that residual symmetries are responsible for a highly symmetric form of $U_{\nu}$. We will parametrize the PMNS matrix in terms of the standard mixing angles and $\mathrm{CP}$ violating phases [15]. The only free parameter here is $\delta$, and analytical expressions for all angles and phases can be obtained (we list them in Appendix A). Experimentally the most well-measured is

$$
\sin \theta_{13}=\frac{\lambda}{3 \sqrt{2}}\left|1+\frac{2 \mathrm{e}^{\mathrm{i} \delta}}{A}\right|+O\left(\lambda^{3}\right) .
$$

Varying $\theta_{13}$ and the Wolfenstein parameters in their experimentally allowed 3(1) $\sigma$ range [16-18] constrains $\delta$ to lie in the interval $\delta / \pi \in\{1.50(1.53), 1.66(1.60)\}$, see Fig. 1. The degeneracy between the sign of $\delta$ and the mixing angles is broken by the recent best-fit value of $\delta_{C P}[18,19]$.

A complete model based on $\mathrm{SO}(10)$ unification with a flavor symmetry ${ }^{1} \mathcal{T}_{13} \cong \mathbb{Z}_{13} \rtimes \mathbb{Z}_{3}$ was put forward in [14] which successfully realizes the asymmetric texture (1). There, cTBM mixing was taken as an assumption, while it was suggested that it may arise from residual symmetries. - If this scheme of mixing indeed originated from a residual symmetry, one

\footnotetext{
${ }^{1}$ See ref. [20-23] for more details on this symmetry.
} 

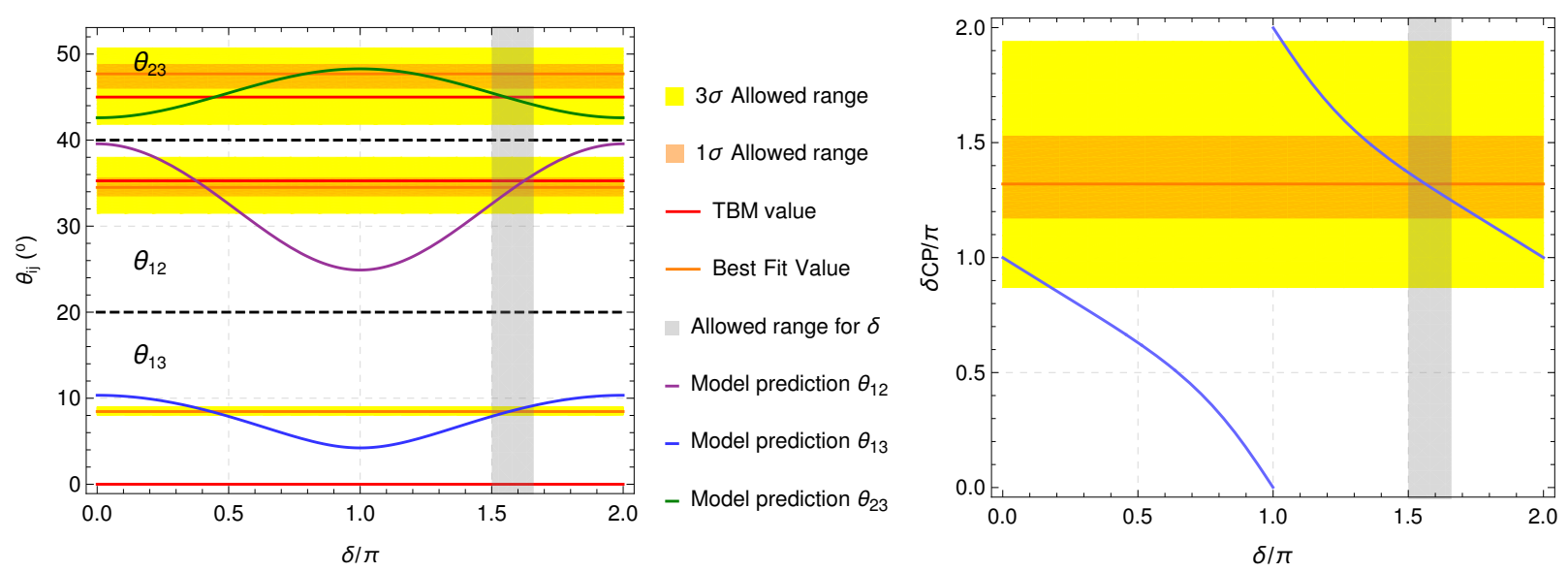

Figure 1: Predictions of asymmetric tri-bi-maximal mixing for lepton mixing angles (left) and Dirac CP violating phase (right) as a function of the internal parameter $\delta$. Experimentally allowed $1(3) \sigma$ regions are shown in orange(yellow). The allowed range for $\delta$ is shaded in gray.

would expect $\delta$ to take a value that is "quantized" in rational fractions of $\pi$. The allowed values of such a quantization intimately depend on the respective residual symmetry group. Exactly because of that, as we will clarify below, some rational values of $\delta / \pi$ are more special than others. We list some of them together with their phenomenological predictions in Tab. I.

In the present note, we derive all possible residual symmetries that realize the cTBM matrix, and clarify how they can be merged into a unified flavor symmetry together with the $\mathcal{T}_{13}$-symmetry of the charged fermion sector. We finish by discussing the phenomenological predictions of the most symmetric scenarios.

\section{SYMMETRY ORIGIN OF ASYMMETRIC TBM MIXING}

\section{A. Residual symmetries of complex TBM mixing}

We start by discussing general residual symmetries of the effective lepton mass terms in the gauge basis

$$
\mathcal{L}_{\text {mass }}=-\bar{\ell}_{\mathrm{L}} M_{\ell} \ell_{\mathrm{R}}-\frac{1}{2} \nu_{\mathrm{L}}^{\mathrm{T}} \mathcal{C} M_{\nu} \nu_{\mathrm{L}}+\text { h.c. },
$$

where $\ell$ denote charged leptons and $\nu$ the neutrinos. The effective neutrino Majorana mass matrix $M_{\nu}$ is symmetric and $\mathcal{C}$ is the charge conjugation matrix. The unitary matrices $U_{\nu, c \ell}$ diagonalize the mass matrices

$$
\begin{aligned}
U_{\nu}^{\mathrm{T}} M_{\nu} U_{\nu} & =\operatorname{diag}\left(m_{1}, m_{2}, m_{3}\right)=: D_{\nu}, \\
U_{c \ell}^{\dagger}\left(M_{\ell} M_{\ell}^{\dagger}\right) U_{c \ell}=\operatorname{diag}\left(m_{e}^{2}, m_{\mu}^{2}, m_{\tau}^{2}\right) & =: D_{\ell} .
\end{aligned}
$$




\begin{tabular}{ccccc}
\hline & $\sin ^{2} \theta_{12}$ & $\sin ^{2} \theta_{13}$ & $\sin ^{2} \theta_{23}$ & $\delta_{C P}$ \\
\hline Best fit (NO) & 0.320 & 0.02160 & 0.547 & $1.32 \pi$ \\
$1 \sigma$ range (NO) & $0.304 \div 0.340$ & $0.02091 \div 0.02243$ & $0.517 \div 0.567$ & $1.17 \pi \div 1.53 \pi$ \\
$3 \sigma$ range (NO) & $0.273 \div 0.379$ & $0.0196 \div 0.0241$ & $0.445 \div 0.599$ & $0.87 \pi \div 1.94 \pi$ \\
$\delta=\mp 9 \pi / 26$ & 0.343 & $\mathbf{0 . 0 2 5 1}$ & $\mathbf{0 . 4 8 5}$ & $\pm 1.25 \pi$ \\
$\delta=\mp 5 \pi / 13$ & 0.331 & $\mathbf{0 . 0 2 3 6}$ & $\mathbf{0 . 4 9 1}$ & $\pm 1.28 \pi$ \\
$\delta=\mp 11 \pi / 26$ & 0.317 & $\mathbf{0 . 0 2 2 0}$ & $\mathbf{0 . 4 9 6}$ & $\pm 1.31 \pi$ \\
$\delta=\mp 6 \pi / 13$ & 0.304 & $\mathbf{0 . 0 2 0 4}$ & $\mathbf{0 . 5 0 2}$ & $\pm 1.34 \pi$ \\
$\delta=\mp \pi / 2$ & 0.289 & $\mathbf{0 . 0 1 8 8}$ & $\mathbf{0 . 5 0 8}$ & $\pm 1.36 \pi$ \\
\hline
\end{tabular}

Table I: Values of the lepton mixing parameters for some special values of the internal parameter $\delta$ for $A$ and $\lambda$ set to their best fit values, together with the experimentally allowed ranges for normal mass ordering (NO). The ranges and best fit points are very similar for inverted ordering (IO). Note that $\delta= \pm 9 \pi / 26$ and $\pm \pi / 2$ are only allowed if the Wolfenstein parameters $A$ and $\lambda$ are also varied.

The diagonal mass matrices $D_{i}$ are all-real. The lepton mixing matrix is defined by

$$
U_{\text {lep }}:=U_{c l}^{\dagger} U_{\nu}
$$

A neutrino mixing matrix for $U_{\nu}$ of the form (2) can be enforced by residual symmetries. ${ }^{2}$ These could be global flavor symmetry transformations [24-28]

$$
\nu_{(x, t)} \mapsto G \nu_{(x, t)}
$$

with some $3 \times 3$ unitary matrices $G$, as well as general CP (GCP) transformations [29] ${ }^{3}$

$$
\nu_{(x, t)} \stackrel{C P}{\longmapsto} \mathrm{i} X \gamma^{0} \mathcal{C} \bar{\nu}_{(-x, t)}^{\mathrm{T}}
$$

with some $3 \times 3$ unitary matrices $X$. Invariance under these transformations requires

$$
G^{\mathrm{T}} M_{\nu} G=M_{\nu} \quad \text { or } \quad X^{\mathrm{T}} M_{\nu} X=M_{\nu}^{*},
$$

respectively. We first focus on pure flavor symmetries and discuss details of possible additional residual GCP symmetries later.

\footnotetext{
2 These "residual symmetries" are not actual symmetries of the full electroweak Lagrangian since they are, in particular, broken by the charged lepton mass terms. Corrections due to this breaking are small scale-dependent effects that we will neglect for the purpose of our discussion.

${ }^{3}$ We have adjusted the unphysical global phase of the $\mathrm{CP}$ transformation to i, such that $M_{\nu}$ is required to be real in a basis where $\mathrm{CP}$ is conserved with a matrix $X=\mathbb{1}$.
} 
Assume that $M_{\nu}$ in the gauge basis is invariant under a symmetry transformation with matrix $G$. It follows that in the mass-diagonal basis (6), $D_{\nu}$ must be invariant under the basis transformed symmetry

$$
E:=U_{\nu}^{\dagger} G U_{\nu}
$$

The invariance condition (11) in this basis translates to

$$
E D_{\nu}=D_{\nu} E^{*}
$$

Assuming non-degenerate and non-zero neutrino masses, the unitary $E$ has to be diagonal and real which means it can only be one of the eight possibilities (uncorrelated signs)

$$
E=\operatorname{diag}( \pm 1, \pm 1, \pm 1)
$$

Under the assumption of a specific form of the mixing matrix $U_{\nu}$, we can translate this back to the gauge basis as $G=U_{\nu} E U_{\nu}^{\dagger}$. Assuming $U_{\nu}=U_{\mathrm{TBM} \delta}$ we find the eight possible residual symmetry transformations in the gauge basis:

$$
\begin{array}{rlrl}
G_{1}^{ \pm} & := \pm \frac{1}{3}\left(\begin{array}{ccc}
-1 & 2 & -2 \mathrm{e}^{-\mathrm{i} \delta} \\
2 & 2 & \mathrm{e}^{-\mathrm{i} \delta} \\
-2 \mathrm{e}^{\mathrm{i} \delta} & \mathrm{e}^{\mathrm{i} \delta} & 2
\end{array}\right), & G_{2}^{ \pm}:= \pm \frac{1}{3}\left(\begin{array}{ccc}
1 & -2 & 2 \mathrm{e}^{-\mathrm{i} \delta} \\
-2 & 1 & 2 \mathrm{e}^{-\mathrm{i} \delta} \\
2 \mathrm{e}^{\mathrm{i} \delta} & 2 \mathrm{e}^{\mathrm{i} \delta} & 1
\end{array}\right) \\
G_{3}^{ \pm}:= \pm\left(\begin{array}{ccc}
-1 & 0 & 0 \\
0 & 0 & \mathrm{e}^{-\mathrm{i} \delta} \\
0 & \mathrm{e}^{\mathrm{i} \delta} & 0
\end{array}\right), & G_{4}^{ \pm}:= \pm\left(\begin{array}{lll}
1 & 0 & 0 \\
0 & 1 & 0 \\
0 & 0 & 1
\end{array}\right) .
\end{array}
$$

As already obvious from (14), and therefore completely independent of the phase $\delta$, these matrices make up for all 8 elements of a group isomorphic to $\mathbb{Z}_{2} \times \mathbb{Z}_{2} \times \mathbb{Z}_{2}$. One of the $\mathbb{Z}_{2}$ factors corresponds to the transformation with a global sign, $G=-\mathbb{1}$, and is automatically a symmetry of the neutrino Majorana mass term in any basis, which is of course well known. Hence, this transformation does not impose any restrictions on $M_{\nu}$. However, we still keep that factor explicit here as it could become meaningful once we unify the residual symmetries of all left-handed leptons into a single group. Furthermore, we note that the remaining $\mathbb{Z}_{2} \times \mathbb{Z}_{2}$ only requires two generators, saying that many of the elements in (15) imply each other. For example, $G_{1}^{+} G_{2}^{+}=G_{3}^{+}$etc.

Consider now the action of possible residual GCP symmetries. In close analogy to above we define

$$
P:=U_{\nu}^{\dagger} X U_{\nu}^{*}
$$

upon which the invariance condition for a GCP transformation in the mass-diagonal basis reads

$$
P D_{\nu}=D_{\nu} P^{*} \text {. }
$$


Under the same assumptions as above this shows that also $P$ can only be one of the eight possibilities listed in (14). Translating this back to the gauge basis via $X=U_{\nu} P U_{\nu}^{\mathrm{T}}$, while assuming the $U_{\mathrm{TBM} \delta}$ form for $U_{\nu}$, one finds the eight possible GCP symmetry transformations:

$$
\begin{array}{rlrl}
X_{1}^{ \pm} & := \pm \frac{1}{3}\left(\begin{array}{ccc}
-1 & 2 & -2 \mathrm{e}^{\mathrm{i} \delta} \\
2 & 2 & \mathrm{e}^{\mathrm{i} \delta} \\
-2 \mathrm{e}^{\mathrm{i} \delta} & \mathrm{e}^{\mathrm{i} \delta} & 2 \mathrm{e}^{2 \mathrm{i} \delta}
\end{array}\right), & X_{2}^{ \pm} & := \pm \frac{1}{3}\left(\begin{array}{ccc}
1 & -2 & 2 \mathrm{e}^{\mathrm{i} \delta} \\
-2 & 1 & 2 \mathrm{e}^{\mathrm{i} \delta} \\
2 \mathrm{e}^{\mathrm{i} \delta} & 2 \mathrm{e}^{\mathrm{i} \delta} & \mathrm{e}^{2 \mathrm{i} \delta}
\end{array}\right), \\
X_{3}^{ \pm}:= \pm\left(\begin{array}{ccc}
-1 & 0 & 0 \\
0 & 0 & \mathrm{e}^{\mathrm{i} \delta} \\
0 & \mathrm{e}^{\mathrm{i} \delta} & 0
\end{array}\right), & X_{4}^{ \pm}:= \pm\left(\begin{array}{ccc}
1 & 0 & 0 \\
0 & 1 & 0 \\
0 & 0 & \mathrm{e}^{2 \mathrm{i} \delta}
\end{array}\right) .
\end{array}
$$

Together, these generate a group $\mathbb{Z}_{2}^{\otimes 4}$ irrespective of the choice of $\delta$. We stress that any combination of two GCP transformations, say with $X_{i}$ and $X_{j}$, induces a flavor symmetry transformation for the neutrinos with $G=-X_{i} X_{j}^{*} \cdot{ }^{4}$ In this way, it is easy to confirm that the union of all GCP transformations, in fact, already contains (i.e. generates) all the eight previously stated flavor symmetries (15). The surplus $\mathbb{Z}_{2}$ factor corresponds to a genuine CP transformation.

\section{B. Unification of complex TBM and $\mathcal{T}_{13}$}

Above the electroweak scale we have $L=\left(\ell_{\mathrm{L}}, \nu_{\mathrm{L}}\right)$, implying that the residual symmetries of left-handed charged leptons and left-handed neutrinos shall originate from a single group globally acting on $L$. Therefore, we will now investigate how the symmetries that give rise to cTBM can be unified with the symmetry group $\mathcal{T}_{13}$, which gives rise to the asymmetric textures of the charged fermion Yukawa couplings resulting in a mixing matrix (1).

In the basis of [14], which is a gauge basis, the $\mathcal{T}_{13}$-symmetric transformation of the charged leptons is generated by the matrices

$$
A:=\left(\begin{array}{ccc}
\rho & 0 & 0 \\
0 & \rho^{3} & 0 \\
0 & 0 & \rho^{9}
\end{array}\right), \quad \text { and } \quad \tilde{B}:=\left(\begin{array}{ccc}
0 & 1 & 0 \\
0 & 0 & 1 \\
1 & 0 & 0
\end{array}\right),
$$

where $\rho^{13}=1$. In the very same basis, possible residual symmetries of the neutrinos are generated by (15) and (18). A common unified flavor symmetry then should contain at least the closure ${ }^{5}$ of some of these elements. Possible outcomes for the unified symmetry will depend on the number of elements that we "add" to $\mathcal{T}_{13}$, and on the value of $\delta$.

\footnotetext{
${ }^{4}$ The additional minus sign here is the usual minus sign that fermions pick up for $(\mathrm{CP})^{2}$ from their Lorentz structure.

${ }^{5}$ Closure here means the matrix group generated by a set of matrix generators, and we will denote it by pointy brackets $\langle\ldots\rangle$.
} 
Before we start this analysis, let us make the following important observation: We can do simultaneous basis transformations of all, charged and neutral, left-handed leptons. These rotations are unphysical, in the sense that they cancel in the physical lepton mixing matrix. Let us perform a simple simultaneous rephasing,

$$
\left(\ell_{\mathrm{L}}^{\prime}, \nu_{\mathrm{L}}^{\prime}\right)=\left(\tilde{P} \ell_{\mathrm{L}}, \tilde{P} \nu_{\mathrm{L}}\right), \quad \text { with } \quad \tilde{P}:=\operatorname{diag}\left(1,1, \mathrm{e}^{-\mathrm{i} \delta}\right)
$$

This removes $\delta$ from the cTBM matrix, as well as from all of its residual symmetries (the outcome is the same as setting $\delta \rightarrow 0$ in (2), (15), and (18)). This restores the usual TBM mixing, alongside its known residual symmetries! However, $\delta$ now appears in $U_{c \ell}$, and $\tilde{B}$. The rephasing does not affect $A$. Of course, the outcome for the unified group is independent of the chosen basis. In the re-phased basis, the only generator that contains $\delta$ is

$$
B:=\tilde{P} \tilde{B} \tilde{P}^{*}=\left(\begin{array}{ccc}
0 & 1 & 0 \\
0 & 0 & \mathrm{e}^{\mathrm{i} \delta} \\
\mathrm{e}^{-\mathrm{i} \delta} & 0 & 0
\end{array}\right)
$$

while $A$, and all the $G$ 's and $X$ 's take their values from above for $\delta \rightarrow 0$. The closure of $\langle A, B\rangle$ is still $\mathcal{T}_{13}$, irrespective of $\delta$. The overall unifying symmetry, however depends on the precise value of $\delta$ and which elements of $G_{i}$ and $X_{i}$ we pick in addition to $A$ and $B$.

Let us briefly mention how family and GCP transformations are combined in practice. We follow the procedure firstly outlined in [30] and collect spinors and their CP conjugates in a single reducible representation, for example $\nu_{\mathrm{L}}(x, t) \oplus-\mathrm{i} \mathcal{C} \nu_{\mathrm{L}}^{*}(-x, t)$. We will suppress the space-time arguments in the following. Family and GCP transformations then act in a single matrix space,

$$
\left(\begin{array}{c}
\nu_{\mathrm{L}} \\
-\mathrm{i} \mathcal{C} \nu_{\mathrm{L}}^{*}
\end{array}\right) \stackrel{G}{\longmapsto}\left(\begin{array}{cc}
G & 0 \\
0 & G^{*}
\end{array}\right)\left(\begin{array}{c}
\nu_{\mathrm{L}} \\
-\mathrm{i} \mathcal{C} \nu_{\mathrm{L}}^{*}
\end{array}\right), \quad \text { or } \quad\left(\begin{array}{c}
\nu_{\mathrm{L}} \\
-\mathrm{i} \mathcal{C} \nu_{\mathrm{L}}^{*}
\end{array}\right) \stackrel{G C P}{\longmapsto}\left(\begin{array}{cc}
0 & X \\
X^{*} & 0
\end{array}\right)\left(\begin{array}{c}
\nu_{\mathrm{L}} \\
-\mathrm{i} \mathcal{C} \nu_{\mathrm{L}}^{*}
\end{array}\right)
$$

respectively. Family and GCP transformations then can be combined simply by matrix multiplication in this enlarged space.

As a first result, note that sets $\langle A, G\rangle$ involving $A$ and elements $G_{1,2}^{ \pm}$or $X_{1,2}^{ \pm}$never close. These, therefore, cannot be unified into a finite group irrespective of $\delta$. They could, however be unified into continuous groups such as $\mathrm{SU}(3)$ or $\mathrm{G}_{2}$; this possibility is left for future work.

On the other hand, elements of $G_{3,4}^{ \pm}$and $X_{3,4}^{ \pm}$form finite groups with $A$ and $B$. We have listed these groups (or their orders, if too large) for certain phenomenologically allowed values of $\delta$ in table II. Coincidentally, groups involving $X_{3}^{ \pm}$often give rise to groups isomorphic to (or at least of the same size than) the extensions by $G_{3}^{ \pm}$. However, it is important to note that those are not the same groups. In particular, they will act differently on the fermions and their mass matrices: For extensions with $G_{3}^{ \pm}, \mathrm{CP}$ conservation is not required and fermions 
transform in a representation $\mathbf{3} \oplus \overline{\mathbf{3}}$. If any GCP transformation is included, however, the fermions will transform in an irreducible 6. Of course, the GCP transformations extend to neutral and charged lepton sectors likewise. Thus, one may wonder whether or how those GCP transformations are consistent with $\mathcal{T}_{13}$ which we observe to be a group of type I. ${ }^{6}$ Type I groups are inconsistent with CP transformations in generic settings. However, this concrete model only features triplet irreps of $\mathcal{T}_{13}$ (cf. tab. I of [14]) for which consistent CP transformations are possible. This situation would drastically change for a model that also contains non-trivial one-dimensional irreps of $\mathcal{T}_{13}$. In such a case, $\mathrm{CP}$ violation with calculable phases would be unavoidable. This would be an interesting starting point for further model building that we do not pursue here.

It is straightforward to check that out of the 12 independent real degrees of freedom in $M_{\nu}, G_{3}^{ \pm}$removes 4 , while $X_{3,4}^{ \pm}$individually each remove 6 . Taken together, $\left\langle G_{3}^{ \pm}, X_{3}^{ \pm}, X_{4}^{ \pm}\right\rangle$ remove 8 degrees of freedom, leaving 4 . We will now focus on this most predictive case. As is already clear from our discussions above, not all generators are needed to generate the maximal possible group $G_{\max }:=\left\langle A, B, G_{3}^{ \pm}, X_{3}^{ \pm}, X_{4}^{ \pm}\right\rangle$. Taking only $\left\langle G_{3}^{ \pm}, X_{3}^{ \pm}\right\rangle,\left\langle G_{3}^{ \pm}, X_{4}^{ \pm}\right\rangle$, or $\left\langle X_{3}^{ \pm}, X_{4}^{ \pm}\right\rangle$in addition to $A$ and $B$ is already sufficient to generate $G_{\max }$. We have ignored the transformations $G_{4}^{ \pm}$here, because they do not lead to constraints on the neutrino mass matrix. Nevertheless, we remark that the according unphysical global $\mathbb{Z}_{2}$ factor (generated by $G_{4}^{-}$) will unavoidably be part of the total flavor symmetry group if any CP transformations are included in the symmetry.

Remarkably, only angles that are quantized in units of 13 or $13 / 2$, i.e. $\delta / \pi=\mathbb{Z} / 26$, lead to groups of acceptable size. The fact that a fraction of 13 gives rise to a small group may not be surprising, given that $\mathcal{T}_{13}$ already contains such a divisor. What is remarkable though, is that putting $\delta$ to $-5(6) \pi / 13$ or $-9(11) \pi / 26$ fits phenomenology well, while at the same time it give rise to the smallest possible finite groups that one can achieve with all those generators - as small as the one obtained for $\delta=0$ or $\delta= \pm \pi / 2$, cf. table II. We will shortly come back to investigate the phenomenology of these particularly symmetric points.

We now comment on these results. The fact that it is impossible to unify the full nontrivial $\mathbb{Z}_{2} \times \mathbb{Z}_{2}$ symmetry of neutrinos with $\mathcal{T}_{13}$ of the charged leptons into a single finite group may seem like a drawback. However, this gives important directions for model building: If one insists on keeping the full $\mathbb{Z}_{2} \times \mathbb{Z}_{2}$ for neutrinos, there is no other way than embedding the residual symmetries directly into a continuous group such as $\mathrm{SU}(3)$ or $\mathrm{G}_{2}$. Quite appealingly,

\footnotetext{
6 "Type I" here refers to the classification of [31]. Type I groups are groups that do not have a classinverting automorphism. This prohibits transformations that simultaneously map all irreps of a group to their respective complex conjugate irreps. Type I groups have no basis with real Clebsch-Gordan coefficients. The complex phases of the Clebsch-Gordan coefficients are known to be a symmetry-based source of explicit CP violation.
} 


\begin{tabular}{|c|c|c|c|}
\hline$\pm \delta / \pi$ & $\left\langle A, B, G_{3}^{ \pm}\right\rangle$ & $\left\langle A, B, X_{3}^{ \pm}\right\rangle$ & $\left\langle A, B, G_{3}^{ \pm}, X_{3}^{ \pm}, X_{4}^{ \pm}\right\rangle$ \\
\hline 0 & $\left(\mathbb{Z}_{13} \times \mathbb{Z}_{13}\right) \rtimes \mathrm{S}_{4}$ & $\left(\mathbb{Z}_{13} \times \mathbb{Z}_{13}\right) \rtimes \mathrm{S}_{4}$ & $\left(\mathbb{Z}_{13} \times \mathbb{Z}_{13}\right) \rtimes\left(\mathrm{S}_{4} \times \mathbb{Z}_{2}\right)$ \\
\hline $1 / 2$ & $\left(\mathbb{Z}_{13} \times \mathbb{Z}_{13}\right) \rtimes \mathrm{S}_{3}$ & $\left(\mathbb{Z}_{13} \times \mathbb{Z}_{13}\right) \rtimes \mathrm{S}_{3}$ & $\left(\mathbb{Z}_{13} \times \mathbb{Z}_{13}\right) \rtimes \mathrm{D}_{12}$ \\
\hline $2 / 5$ & $\mathcal{O}(101,400)$ & $\mathcal{O}(101,400)$ & $\mathcal{O}(202,800)$ \\
\hline $3 / 7$ & $\mathcal{O}(198,744)$ & $\mathcal{O}(198,744)$ & $\mathcal{O}(397,488)$ \\
\hline $3 / 8$ & $\mathcal{O}(64,896)$ & $\mathcal{O}(64,896)$ & $\mathcal{O}(129,792)$ \\
\hline $4 / 9$ & $\mathcal{O}(109,512)$ & $\mathcal{O}(328,536)$ & $\mathcal{O}(657,072)$ \\
\hline $5 / 11$ & $\mathcal{O}(490,776)$ & $\mathcal{O}(490,776)$ & $\mathcal{O}(981,552)$ \\
\hline $5 / 12$ & $\mathcal{O}(48,672)$ & $\mathcal{O}(146,016)$ & $\mathcal{O}(292,032)$ \\
\hline $5 / 13 ; 6 / 13$ & $\left(\mathbb{Z}_{13} \times \mathbb{Z}_{13}\right) \rtimes \mathrm{S}_{4}$ & $\left(\mathbb{Z}_{13} \times \mathbb{Z}_{13}\right) \rtimes \mathrm{S}_{4}$ & $\left(\mathbb{Z}_{13} \times \mathbb{Z}_{13}\right) \rtimes\left(\mathrm{S}_{4} \times \mathbb{Z}_{2}\right)$ \\
\hline $5 / 14$ & $\mathcal{O}(49,686)$ & $\mathcal{O}(49,686)$ & $\mathcal{O}(99,372)$ \\
\hline $7 / 18$ & $\mathcal{O}(27,378)$ & $\mathcal{O}(82,134)$ & $\mathcal{O}(164,268)$ \\
\hline $9 / 26 ; 11 / 26$ & $\left(\mathbb{Z}_{13} \times \mathbb{Z}_{13}\right) \rtimes \mathrm{S}_{3}$ & $\left(\mathbb{Z}_{13} \times \mathbb{Z}_{13}\right) \rtimes \mathrm{S}_{3}$ & $\left(\mathbb{Z}_{13} \times \mathbb{Z}_{13}\right) \rtimes \mathrm{D}_{12}$ \\
\hline$\{14 ; 16 ; 17 ; 19\} / 39$ & $\mathcal{O}(12,168)$ & $\mathcal{O}(36,504)$ & $\mathcal{O}(73,008)$ \\
\hline$\{29 ; 31 ; 35 ; 37\} / 78$ & $\mathbb{Z}_{3} \times\left(\mathbb{Z}_{13} \times \mathbb{Z}_{13}\right) \rtimes \mathrm{S}_{3}$ & $\mathcal{O}(9,126)$ & $\mathcal{O}(18,252)$ \\
\hline
\end{tabular}

Table II: Possibilities for unified flavor symmetry groups (or their order, if too large) for fractional values of $\delta$ in the phenomenologically allowed interval $\delta / \pi \in\{1.50,1.66\}$. Other values for $\delta$ lead to groups even larger than the ones listed, with orders easily exceeding $\mathcal{O}\left(10^{5}\right)$. Groups $\left\langle A, B, G_{1,2}^{ \pm}\right\rangle$ and $\left\langle A, B, X_{1,2}^{ \pm}\right\rangle$are $\mathcal{O}(\infty)$ irrespective of the value of $\delta$. Groups are stated modulo the unphysical global $\mathbb{Z}_{2}$ factor generated by $G_{4}^{-}$. These results have been obtained with GAP [32].

this would point to the fact that there might be no intermediate scales, or complicated finite groups, involved between the flavor breaking scale and the low scale.

Alternatively, one may be fine with just keeping those parts of the residual neutrino symmetries that form finite groups with $\mathcal{T}_{13}$. Depending on the choice of $\delta$, the unified symmetry then will be one of the groups listed in table II. However, even in the "maximally finitesymmetric case" $G_{\max }$, there are four independent degrees of freedom in the neutrino mass matrix. Somewhat ironically this means that by fixing $\delta$ to a geometric value, we are forced to liberate another degree of freedom elsewhere. This implies that there will, in general, be model dependent corrections to the complex-TBM form which are not constrained by the residual symmetries. We stress that those corrections do not have to be large, as is known from explicit models. For example, in $[33,34]$ it happens that TBM-mixing is realized at leading order despite the fact that there is only a $\mathbb{Z}_{2}$ residual symmetry, while in [20] leading order TBM appears even in the complete absence of residual symmetries.

Finally, the third and most radical alternative would be to revisit the charged lepton sector and search for symmetries beyond $\mathcal{T}_{13}$ which are able to produce asymmetric textures, while 
being able to incorporate the full residual symmetries of the neutrinos.

\section{PREDICTIONS OF THE MAXIMALLY SYMMETRIC CASE}

We now focus on the maximally finite-symmetric case with unified flavor symmetry $G_{\max }$ and point out phenomenological consequences. Taking $G_{\max }$ to be one of the two smallest possible unified flavor groups, while requiring phenomenologically allowed mixing and CP violation, $\delta$ is restricted to take the values $\delta / \pi \times(-26)=\{9,10,11,12\}$. For any value of $\delta$, the neutrino mass matrix that respects the residual flavor symmetry and GCP transformations $\left\langle G_{3}^{ \pm}, X_{3}^{ \pm}, X_{4}^{ \pm}\right\rangle$, takes the four-parameter form

$$
M_{\nu}=\left(\begin{array}{ccc}
a & b & -b \mathrm{e}^{-\mathrm{i} \delta} \\
b & c & d \mathrm{e}^{-\mathrm{i} \delta} \\
-b \mathrm{e}^{-\mathrm{i} \delta} & d \mathrm{e}^{-\mathrm{i} \delta} & c \mathrm{e}^{-2 \mathrm{i} \delta}
\end{array}\right) .
$$

Here, $a, b, c$, and $d$ are real parameters with dimensions of mass, and we go back to use our initial basis of eqs. $(1,2)$. The neutrino mixing matrix then is of the form ${ }^{7}$

$$
U_{\nu}=U_{\mathrm{TBM} \delta} O_{12}(\gamma)
$$

where $\gamma$ is one combination of the free parameters that corresponds to the angle of an orthogonal rotation $O_{12}(\gamma)$ in the 12-block. The remaining three free parameters correspond to the neutrino masses. Hence, also all column permutations of Eq. (24) would diagonalize Eq. (23) with a viable mass spectrum. However, $1 \leftrightarrow 2$ permutations, here, give rise to physically identical results (recall that $\theta_{12}$ can be adjusted by a free parameter). By constrast, the other permutations are phenomenologically excluded since they cannot fit $\theta_{13}$ and $\theta_{23}$ at the same time. This implies that $\theta_{13}$ and $\theta_{23}$ remain fixed at their values of the original asymmetric texture, already stated in bold in table I. In contrast, $\theta_{12}$ and all CP violating Dirac and Majorana phases become functions of the single free parameter $\gamma$. Hence, we can trade $\gamma$ for $\theta_{12}$ and the corresponding correlations are shown graphically in Fig. 2. To display Majorana phases we use the so-called symmetrical parametrization [36, 37] which is related to the standard parametrization [15] via $\alpha \equiv \alpha_{21} \equiv-2 \phi_{12}, \beta \equiv \alpha_{31} \equiv-2 \phi_{13}$, and $\delta_{C P} \equiv \phi_{13}-\phi_{12}-\phi_{23}$. The observational constraints on $\theta_{12}$ and $\delta_{C P}$ constrain $\gamma$ (for example, for $\delta / \pi=-11 / 26)$ to the range $\gamma \in\{-0.051,0.067\}$; this fits well with the expectation of it being a small perturbation. Our model assumes neutrinos with effective Majorana masses. Therefore, neutrinoless double beta decay is expected to be present. Since $\theta_{13}$ and $\theta_{23}$ are fixed while the CP phases are all strongly correlated this model has definite predictions

\footnotetext{
7 This can be shown using the analysis tools of [35]. To convince oneself, one may also apply a rotation with $U_{\mathrm{TBM} \delta}$ to $M_{\nu}$ of eq. (23) and see that the missing step to a diagonalization is a 12 rotation.
} 

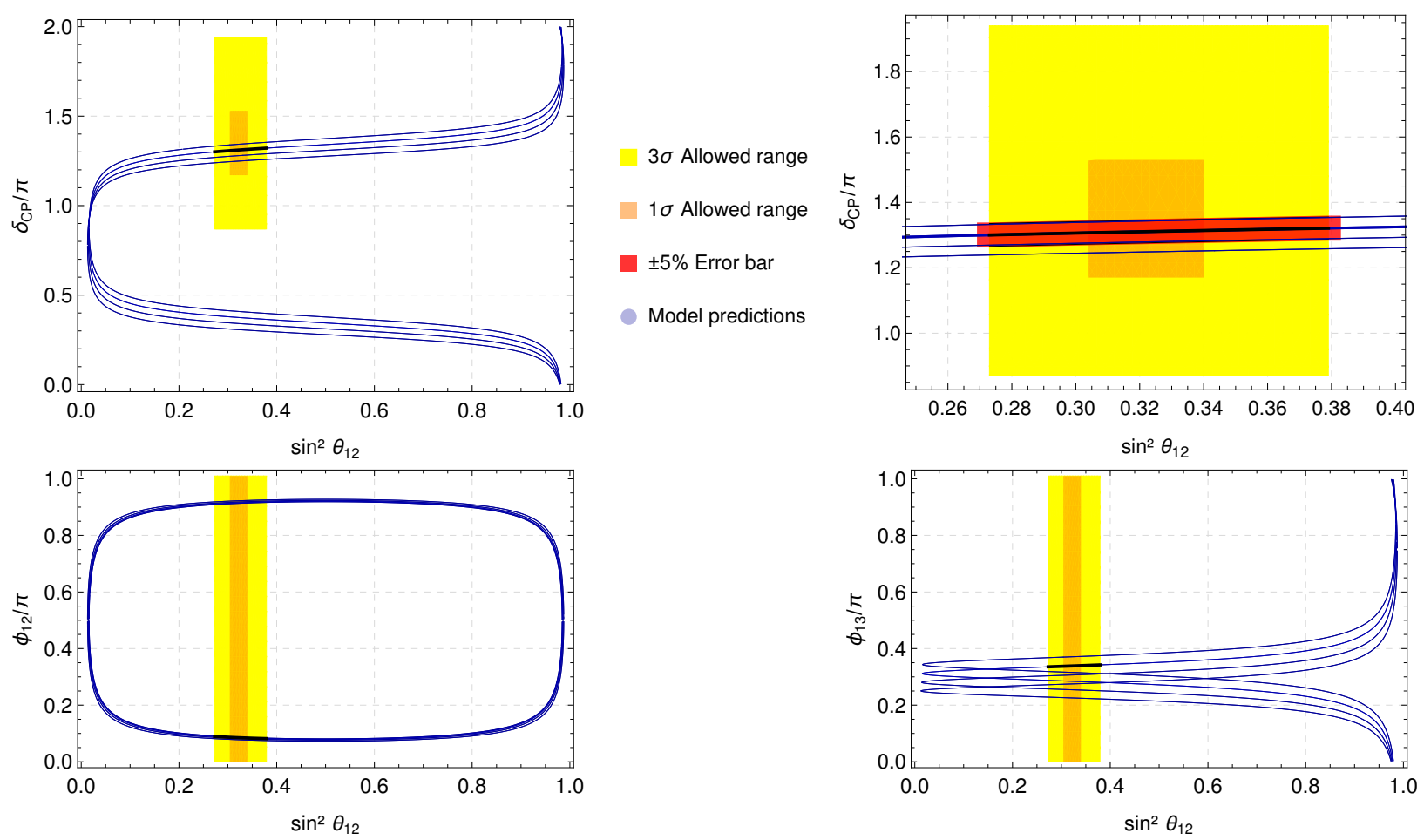

Figure 2: Prediction for CP violating phases in correlation with $\theta_{12}$ (blue lines) in the maximally finite-symmetric case, for fixed values of $\delta / \pi \times(-26)=\{9,10,11,12\}$ (the ordering is from bottom to top in the important regions of the plots). Highlighted in black is the range $\gamma \in\{-0.051,0.067\}$ for $\delta / \pi=-11 / 26$. On the top right is a zoom of the top left plot. There we show a $\pm 5 \%$ error bar for illustration on the $\delta / \pi=-11 / 26$ line, cf. the related discussion in the text. The related predictions for $\theta_{13}$ and $\theta_{23}$ are numbers, already stated in bold in table I.

for neutrinoless double beta decay. Fixing the lightest neutrino mass, the effective electron Majorana mass $m_{e e}$ only depends on one parameter. We show this in Fig. 3. Clearly, this is a scenario in which also the case of normal mass ordering can be excluded by non-observation of neutrinoless double beta decay.

Altogether, the parameters obtained in this scheme for $\delta / \pi=-11 / 26$ and $\gamma=0$ are

$$
\begin{array}{lll}
s_{\theta_{13}}^{2} \approx 0.0220, & s_{\theta_{12}}^{2} \approx 0.317, & s_{\theta_{23}}^{2} \approx 0.496, \\
\delta_{C P} \approx 1.31 \pi, & \phi_{12} \approx 0.09 \pi, & \phi_{13} \approx 0.34 \pi \\
\left.m_{e e}^{\mathrm{NO}}\right|_{m_{\text {lightest } \rightarrow 0} \approx 2.9 \times 10^{-3} \mathrm{eV},}, & \left.m_{e e}^{\mathrm{IO}}\right|_{m_{\text {lightest } \rightarrow 0}} \approx 4.8 \times 10^{-2} \mathrm{eV} . &
\end{array}
$$

These coincide with the values given in Tab. I for $\delta=-11 \pi / 26$, since $\gamma=0$. Varying the free parameter $\gamma$ will result in a modification of $\theta_{12}$ and the CPV phases while $\theta_{13}$ and $\theta_{23}$ preserve their values. The effect of such a variation is negligible at the current accuracy. For example, bringing $\theta_{12}$ to its current best fit value requires $\gamma \approx 4 \times 10^{-4}$.

Let us now comment on the precision of the predictions in our scenario. First and 


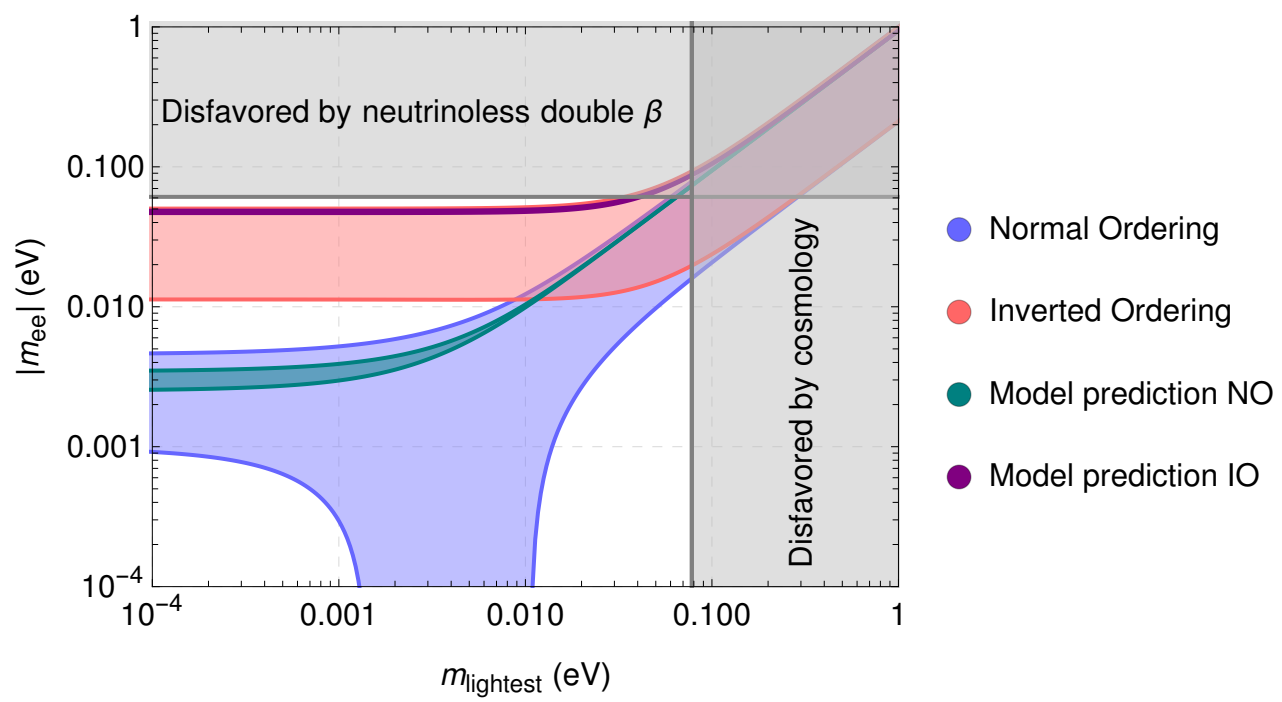

Figure 3: Effective electron neutrino Majorana mass as a function of the lightest neutrino mass. Varying the mixing parameters in their $3 \sigma$ range leads to the blue(red) region for normal(inverted) ordering. The predictions of asymmetric tri-bi-maximal mixing with $\delta / \pi=-11 / 26$, as it may originate from a unified discrete flavor symmetry $\left(\mathbb{Z}_{13} \times \mathbb{Z}_{13}\right) \rtimes \mathrm{D}_{12}$, are shown in green (NO) and purple (IO). We also show exclusion regions from Planck [38] and KamLAND-Zen [39].

foremost, we have neglected terms of $\mathcal{O}\left(\lambda^{3}\right)$ which roughly amounts to a $5 \%$ uncertainty on the mixing angles. Also the values of $A$ and $\lambda$ itself are subject to uncertainties. Furthermore, one would expect corrections to the residual symmetry pattern originating from the RGE evolution below the flavor breaking scale. Assuming normal mass ordering and no new physics thresholds below the flavor breaking scale, the effect on mixing angles and phases is generically of the order $\mathcal{O}\left(10^{-5}\right)$ [40] and, therefore, completely negligible. For illustration, we show a $\pm 5 \%$ uncertainty band in figure 2 . The current level of precision makes it impossible even theoretically to differentiate between the predictions of neighboring values of the fine-grained quantized angle $\delta$.

\section{SUMMARY}

Recently, an SU(5) based model of grand unification with $\mathcal{T}_{13}$ flavor symmetry was suggested by $[13,14]$. This very special flavor symmetry provides a connection between quark and charged lepton sectors, providing a symmetry origin for the so-called asymmetric texture of charged lepton mixing, eq. (1). Together with the complex tri-bi-maximal mixing matrix for neutrinos, shown in (2), this gives rise to a phenomenologically viable one-parameter $(\delta)$ fit to lepton mixing and CP violation, see Fig. 1.

Here, we have studied how complex tri-bi-maximal mixing can be enforced by residual 
flavor and/or generalized CP symmetries of neutrinos, and how those symmetries can be unified with the $\mathcal{T}_{13}$ flavor symmetry of the charged fermions. Unification of the maximal residual symmetry of (2) with $\mathcal{T}_{13}$ requires unified flavor symmetry groups of infinite order such as $S U(3)$ or $G_{2}$. However, if only parts of the maximal residual symmetry are imposed it is possible to unify them with $\mathcal{T}_{13}$ to a finite unified flavor symmetry. Reasonably small finite unified flavor groups are only obtained if $2 \delta$ is quantized in multiples of $\pi / 13$. In particular, $\delta / \pi=-11 / 26$ provides a good fit to the data while it can originate from the smallest possible unified flavor group $\left(\mathbb{Z}_{13} \times \mathbb{Z}_{13}\right) \rtimes \mathrm{D}_{12}$.

However, even though $\delta$ can be fixed by residual symmetries that form finite groups with $\mathcal{T}_{13}$, none of these groups is maximally restrictive on the neutrino mixing matrix. Thus, the residual symmetries allow for one additional unconstrained parameter, $\gamma$, which corresponds to a rotation in the 1-2 space. Nontheless, since $\gamma$ is observationally constrained to be a small perturbation, sharp predictions for CP violating phases and neutrinoless double-beta decay are possible, see eq. (25) and Figs. 2 and 3.

This model provides a compelling target for future experiments. It could be fully excluded by a more precise measurement of $\theta_{23}$ or non-observation of neutrinoless double-beta decay - even for normal neutrino mass ordering.

\section{Acknowledgments}

SCC would like to thank the Max-Planck-Institute for Nuclear Physics in Heidelberg for hospitality during his visit, where this work was initiated. The work of SCC is supported by the Spanish grants SEV-2014-0398, FPA2017-85216-P (AEI/FEDER, UE) and PROMETEO/2018/165 (Generalitat Valenciana), Red Consolider MultiDark FPA2017-90566-REDC and BES-2016-076643.

\section{Appendix A: Analytic expressions}

Analytic expressions for the mixing angles are given by eq. (4) and

$$
\begin{aligned}
\sin \theta_{12} & =\frac{1}{\sqrt{3}}-\frac{\lambda}{3 \sqrt{3} A}(A-2 \cos \delta)-\frac{\lambda^{2}}{36 \sqrt{3} A^{2}}\left(A^{2}-4 A \cos \delta+4 \cos 2 \delta\right)+\mathcal{O}\left(\lambda^{3}\right) \\
\sin \theta_{23} & =\frac{1}{\sqrt{2}}-\frac{\lambda^{2}}{36 \sqrt{2} A}\left[A^{2}-4+4\left(A+9 A^{3}\right) \cos \delta\right]+\mathcal{O}\left(\lambda^{3}\right)
\end{aligned}
$$


The CP-odd Jarlskog-Greenberg invariant and the two Majorana invariants are given by

$$
\begin{aligned}
J & =\frac{\sin \delta}{9 A}\left(\lambda-\frac{\lambda^{2}}{3}\right)+\mathcal{O}\left(\lambda^{3}\right), \\
I_{1} & =\frac{4 \sin \delta}{9 A}\left[\lambda-\frac{\lambda^{2}}{6 A^{2}}(A-2 \cos \delta)\right]+\mathcal{O}\left(\lambda^{3}\right), \\
I_{2} & =\frac{4 \sin \delta}{27 A^{2}} \lambda^{2}(A+2 \cos \delta)+\mathcal{O}\left(\lambda^{3}\right) .
\end{aligned}
$$

The Dirac and Majorana phases are given by

$$
\begin{aligned}
\sin \delta_{C P}= & \frac{2 \sin \delta}{\left|A+2 \mathrm{e}^{\mathrm{i} \delta}\right|}\left[1-\frac{\lambda}{6 A}(A+2 \cos \delta)\right. \\
& \left.+\frac{\lambda^{2}}{36 A^{2}}\left(9-A^{2}+36 A^{4}+8\left(A+9 A^{3}\right) \cos \delta+11 \cos 2 \delta\right)\right]+\mathcal{O}\left(\lambda^{3}\right), \\
\sin \alpha= & \sin \left(-2 \phi_{12}\right)=\frac{2 \sin \delta}{A}\left[\lambda+\frac{\lambda^{2}}{6 A}(A-2 \cos \delta)\right]+\mathcal{O}\left(\lambda^{3}\right), \\
\sin \beta= & \sin \left(-2 \phi_{13}\right)=\frac{4 \sin \delta}{\left|A+2 \mathrm{e}^{\mathrm{i} \delta}\right|^{2}}\left[(A+2 \cos \delta)+\frac{\lambda}{6 A}\left(A^{2}+4 A \cos \delta+4 \cos 2 \delta\right)\right. \\
& \left.-\frac{\lambda^{2}}{36 A^{2}}\left(A^{3}+2 A^{2} \cos \delta-4 A \cos 2 \delta-8 \cos 3 \delta\right)\right]+\mathcal{O}\left(\lambda^{3}\right) .
\end{aligned}
$$

[1] A. Yu. Smirnov, "Neutrinos: '...Annus mirabilis'," in Proceedings of the 2nd NO-VE International Workshop on Neutrino Oscillations: Venice, December 3-5, 2003, pp. 1-21. 2004. arXiv:hep-ph/0402264 [hep-ph].

[2] H. Minakata and A. Yu. Smirnov, "Neutrino mixing and quark-lepton complementarity," Phys. Rev. D70 (2004) 073009, arXiv:hep-ph/0405088 [hep-ph].

[3] M. Raidal, "Relation between the neutrino and quark mixing angles and grand unification," Phys. Rev. Lett. 93 (2004) 161801, arXiv:hep-ph/0404046 [hep-ph].

[4] P. F. Harrison, D. H. Perkins, and W. G. Scott, "Tri-bimaximal mixing and the neutrino oscillation data," Phys. Lett. B530 (2002) 167, arXiv:hep-ph/0202074 [hep-ph].

[5] P. F. Harrison and W. G. Scott, "Symmetries and generalizations of tri - bimaximal neutrino mixing," Phys. Lett. B535 (2002) 163-169, arXiv:hep-ph/0203209 [hep-ph].

[6] A. Datta, L. Everett, and P. Ramond, "Cabibbo haze in lepton mixing," Phys. Lett. B620 (2005) 42-51, arXiv:hep-ph/0503222 [hep-ph].

[7] L. L. Everett, "Viewing lepton mixing through the Cabibbo haze," Phys. Rev. D73 (2006) 013011, arXiv:hep-ph/0510256 [hep-ph].

[8] J. Kile, M. J. Pérez, P. Ramond, and J. Zhang, "Majorana Physics Through the Cabibbo Haze," JHEP 02 (2014) 036, arXiv:1311.4553 [hep-ph]. [Erratum: JHEP11,158(2014)]. 
[9] C. Giunti and M. Tanimoto, "Deviation of neutrino mixing from bimaximal," Phys. Rev. D 66 (2002) 053013, arXiv:hep-ph/0207096.

[10] P. Frampton, S. Petcov, and W. Rodejohann, "On deviations from bimaximal neutrino mixing," Nucl. Phys. B 687 (2004) 31-54, arXiv:hep-ph/0401206.

[11] S. Goswami, S. T. Petcov, S. Ray, and W. Rodejohann, "Large $|U(e 3)|$ and Tri-bimaximal Mixing," Phys. Rev. D 80 (2009) 053013, arXiv:0907.2869 [hep-ph].

[12] J. Kile, M. J. Pérez, P. Ramond, and J. Zhang, " $\theta_{13}$ and the flavor ring," Phys. Rev. D90 no. 1, (2014) 013004, arXiv:1403.6136 [hep-ph].

[13] M. H. Rahat, P. Ramond, and B. Xu, "Asymmetric tribimaximal texture," Phys. Rev. D98 no. 5, (2018) 055030, arXiv:1805.10684 [hep-ph].

[14] M. J. Pérez, M. H. Rahat, P. Ramond, A. J. Stuart, and B. Xu, "Stitching an asymmetric texture with $\mathcal{T}_{13} \times \mathcal{Z}_{5}$ family symmetry," Phys. Rev. D100 no. 7, (2019) 075008, arXiv:1907.10698 [hep-ph].

[15] Particle Data Group Collaboration, M. Tanabashi et al., "Review of Particle Physics," Phys. Rev. D98 no. 3, (2018) 030001.

[16] P. F. de Salas et al., "Status of neutrino oscillations 2018: $3 \sigma$ hint for normal mass ordering and improved CP sensitivity," Phys. Lett. B782 (2018) 633-640, arXiv:1708.01186 [hep-ph]. http://globalfit.astroparticles.es/.

[17] Valencia-Globalfit. http://globalfit.astroparticles.es/, 2018.

[18] P. F. de Salas, S. Gariazzo, O. Mena, C. A. Ternes, and M. Tórtola, "Neutrino Mass Ordering from Oscillations and Beyond: 2018 Status and Future Prospects," arXiv:1806.11051 [hep-ph].

[19] I. Esteban, M. C. Gonzalez-Garcia, A. Hernandez-Cabezudo, M. Maltoni, and T. Schwetz, "Global analysis of three-flavour neutrino oscillations: synergies and tensions in the determination of $\theta_{23}, \delta_{C P}$, and the mass ordering," JHEP 01 (2019) 106, arXiv:1811.05487 [hep-ph].

[20] G.-J. Ding, "Tri-Bimaximal Neutrino Mixing and the $T_{13}$ Flavor Symmetry," Nucl. Phys. B853 (2011) 635-662, arXiv:1105.5879 [hep-ph].

[21] C. Hartmann and A. Zee, "Neutrino Mixing and the Frobenius Group T13," Nucl. Phys. B853 (2011) 105-124, arXiv:1106.0333 [hep-ph].

[22] Y. Kajiyama and H. Okada, "T(13) Flavor Symmetry and Decaying Dark Matter," Nucl. Phys. B848 (2011) 303-313, arXiv:1011.5753 [hep-ph].

[23] C. Hartmann, "The Frobenius group T13 and the canonical see-saw mechanism applied to neutrino mixing," Phys. Rev. D85 (2012) 013012, arXiv:1109.5143 [hep-ph].

[24] E. Ma, "Neutrino mass matrix from S(4) symmetry," Phys. Lett. B632 (2006) 352-356, arXiv:hep-ph/0508231 [hep-ph].

[25] C. S. Lam, "Symmetry of Lepton Mixing," Phys. Lett. B656 (2007) 193-198, 
arXiv:0708.3665 [hep-ph].

[26] A. Blum, C. Hagedorn, and M. Lindner, "Fermion Masses and Mixings from Dihedral Flavor Symmetries with Preserved Subgroups," Phys. Rev. D77 (2008) 076004, arXiv:0709.3450 [hep-ph].

[27] W. Grimus, L. Lavoura, and P. O. Ludl, "Is S(4) the horizontal symmetry of tri-bimaximal lepton mixing?," J. Phys. G36 (2009) 115007, arXiv:0906.2689 [hep-ph].

[28] D. Hernandez and A. Yu. Smirnov, "Lepton mixing and discrete symmetries," Phys. Rev. D86 (2012) 053014, arXiv:1204.0445 [hep-ph].

[29] F. Feruglio, C. Hagedorn, and R. Ziegler, "Lepton Mixing Parameters from Discrete and CP Symmetries," JHEP 07 (2013) 027, arXiv:1211.5560 [hep-ph].

[30] M. Holthausen, M. Lindner, and M. A. Schmidt, "CP and Discrete Flavour Symmetries," JHEP 04 (2013) 122, arXiv:1211.6953 [hep-ph].

[31] M.-C. Chen, M. Fallbacher, K. T. Mahanthappa, M. Ratz, and A. Trautner, "CP Violation from Finite Groups," Nucl. Phys. B883 (2014) 267-305, arXiv:1402.0507 [hep-ph].

[32] The GAP Group, GAP - Groups, Algorithms, and Programming, Version 4.10.0, 2018. https://www.gap-system.org.

[33] G. Altarelli and F. Feruglio, "Tri-bimaximal neutrino mixing from discrete symmetry in extra dimensions," Nucl. Phys. B720 (2005) 64-88, arXiv:hep-ph/0504165 [hep-ph].

[34] G. Altarelli and F. Feruglio, "Tri-bimaximal neutrino mixing, A(4) and the modular symmetry," Nucl. Phys. B741 (2006) 215-235, arXiv:hep-ph/0512103 [hep-ph].

[35] P. Chen, S. Centelles Chuliá, G.-J. Ding, R. Srivastava, and J. W. F. Valle, "CP symmetries as guiding posts: revamping tri-bi-maximal mixing. Part I," JHEP 03 (2019) 036, arXiv:1812.04663 [hep-ph].

[36] J. Schechter and J. W. F. Valle, "Neutrino Masses in SU(2) x U(1) Theories," Phys. Rev. D22 (1980) 2227.

[37] W. Rodejohann and J. W. F. Valle, "Symmetrical Parametrizations of the Lepton Mixing Matrix," Phys. Rev. D84 (2011) 073011, arXiv:1108.3484 [hep-ph].

[38] Planck Collaboration, N. Aghanim et al., "Planck 2018 results. VI. Cosmological parameters," arXiv:1807.06209 [astro-ph.C0].

[39] KamLAND-Zen Collaboration, A. Gando et al., "Search for Majorana Neutrinos near the Inverted Mass Hierarchy Region with KamLAND-Zen," Phys. Rev. Lett. 117 no. 8, (2016) 082503, arXiv: 1605.02889 [hep-ex]. [Addendum: Phys. Rev.

Lett.117,no.10,109903(2016)].

[40] S. Antusch, J. Kersten, M. Lindner, and M. Ratz, "Running neutrino masses, mixings and CP phases: Analytical results and phenomenological consequences," Nucl. Phys. B674 (2003) 401-433, arXiv:hep-ph/0305273 [hep-ph]. 